\title{
MAXIMAL NUMERICAL RANGE AND QUADRATIC ELEMENTS IN A $C^{*}$-ALGEBRA
}

\author{
E. H. Benabdi, M. BarraA, M. K. Chraibi And A. Baghdad*
}

Abstract. In this paper, we give a description of the maximal numerical range of a hyponormal element and a characterization of a normaloid element in a $C^{*}$-algebra. We also give an explicit formula for the maximal numerical range of a quadratic operator acting on a complex Hilbert space. As a consequence, we determine the maximal numerical range of a rank-one operator.

Mathematics subject classification (2020): Primary 46L05; Secondary 47A12, 47A63.

Keywords and phrases: Quadratic operator, $C^{*}$-algebra, numerical range, maximal numerical range.

\section{REFERENCES}

[1] A. Abu-Omar And P. Y. WU, Scalar approximants of quadratic operators with applications, Oper. Matrices, 12 (1) (2018), 253-262.

[2] B. Aupetit, A primer on spectral theory, New York (NY): Springer Verlag (1991).

[3] A. BAGHDAD AND M. C. KAADOUD, On the maximal numerical range of a hyponormal operator, Oper. Matrices, 13 (4) (2019), 1163-1171.

[4] F. F. Bons ALl AND J. DunCAN, Numerical ranges of operators on normed spaces and of elements of normed algebras, London-New York: Cambridge University Press, (1971), (London mathematical society lecture note series; 2 ).

[5] F. F. Bonsall and J. Duncan, Numerical ranges II, New York-London: Cambridge University Press; (1973), (London mathematical society lecture notes series; 10).

[6] J. T. ChAN AND K. ChAn, An observation about normaloid operators, Oper. Matrices, 11 (3) (2017), $885-890$.

[7] C. K. Fong, On the essential maximal numerical range, Acta Sci. Math., 41 (1979), 307-315.

[8] K. E. GUSTAFSON AND D. K. M. RAO, Numerical range: the field of values of linear operators and matrices, Springer, New York, Inc: 1997.

[9] P. R. Halmos, Hilbert space problem book, New York: Van Nostrand (1967).

[10] A. N. HAMED AND I. M. SPITKOVSKY, On the maximal numerical range of some matrices, Electron J. Linear Algebra, 34 (2018), 288-303.

[11] G. Ji, N. LIU AND Z. E. LI, Essential numerical range and maximal numerical range of the Aluthge transform, Linear Multilinear Algebra, 55 (4) (2007), 315-322.

[12] D. N. Kingangi, On Norm of Elementary Operator: An Application of Stampfli's Maximal Numerical Range, Pure Appl. Math. J., 7 (1) (2018), 6-10.

[13] C. S. KubRusLy, Spectral Theory of Bounded Linear Operators, Birkhäuser (2020).

[14] J. Rooin, S. Karami And M. G. Aghideh, A new approach to numerical radius of quadratic operators, Ann. Funct. Anal., 11 (2020), 879-896.

[15] I. M. Spitkovs Ky, A note on the maximal numerical range, Oper. Matrices, 13(3) (2019), 601-605.

[16] J. G. STAMPFLi AND J. P. Williams, Growth condition and the numerical range in Banach algebra, Tohoku Math. J. 20 (1968), 417-424.

[17] J. G. Stampfli, The norm of derivation, Pacific J. Math., 33 (1970), 737-747.

[18] S. H. Tso AND P. Y. Wu, Matricial ranges of quadratic operators, Rocky Mountain J. Math., 29 (1999), 1139-1152. 